\title{
Existence results for fractional order differential equation with nonlocal Erdélyi-Kober and generalized Riemann-Liouville type integral boundary conditions at resonance
}

Qiao Sun', Shuman Meng ${ }^{1}$ and Yujun Cui ${ }^{*}$

"Correspondence:

cyj720201@163.com

${ }^{2}$ State Key Laboratory of Mining

Disaster Prevention and Control

Co-founded by Shandong Province

and the Ministry of Science and

Technology, Shandong University of

Science and Technology, Qingdao,

P.R. China

Full list of author information is

available at the end of the article

\begin{abstract} value problem

$$
\begin{aligned}
& { }^{c} D^{q} x(t)=f\left(t, x(t), x^{\prime}(t)\right), \quad t \in[0, T], 1<q \leq 2, \\
& x(0)=\left.\alpha\right|_{\eta} ^{\gamma, \delta} x(\zeta), \quad x(T)=\beta^{\rho} p_{x} x(\xi),
\end{aligned}
$$
\end{abstract}

In this paper, we discuss a nonlinear fractional order boundary value problem with nonlocal Erdélyi-Kober and generalized Riemann-Liouville type integral boundary conditions. By using Mawhin continuation theorem, we investigate the existence of solutions of this boundary value problem at resonance. It is shown that the boundary

has at least one solution under some suitable conditions, where $\alpha, \beta \in \mathbb{R}, 0<\zeta, \xi<T$.

Keywords: Boundary value problem; Resonance; Integral conditions

\section{Introduction}

In this paper, we intend to discuss the following boundary value problem at resonance:

$$
\left\{\begin{array}{l}
{ }^{c} D^{q} x(t)=f\left(t, x(t), x^{\prime}(t)\right), \quad t \in[0, T], \\
x(0)=\alpha I_{\eta}^{\gamma, \delta} x(\zeta), \quad x(T)=\beta^{\rho} I^{p} x(\xi), \quad 0<\zeta, \eta \leq T,
\end{array}\right.
$$

where ${ }^{c} D^{q}$ is the Caputo fractional derivative of order $1<q \leq 2, I_{\eta}^{\gamma, \delta}$ is a Erdélyi-Kober type integral of order $\delta>0$ with $\eta>0$ and $\gamma \in \mathbb{R},{ }^{\rho} I^{p}$ denotes the generalized RiemannLiouville type integral of order $p>0, \rho>0$, and $\alpha, \beta \in \mathbb{R}$.

Boundary value problems at resonance have aroused people's interest these days (see [5, $6,8,9,14,16,17,19-21,25,29-33,35,40,41,43]$ ). For instance, in [17], Jiang and Qiu studied the existence of solutions for the following $(k, n-k)$ conjugate boundary value problem at resonance:

$$
(-1)^{n-k} y^{(n)}(t)=f\left(t, y(t), y^{\prime}(t), \ldots, y^{(n-1)}(t)\right), \quad t \in[0,1],
$$

(0) The Author(s) 2018. This article is distributed under the terms of the Creative Commons Attribution 4.0 International License (http://creativecommons.org/licenses/by/4.0/), which permits unrestricted use, distribution, and reproduction in any medium, provided you give appropriate credit to the original author(s) and the source, provide a link to the Creative Commons license, and indicate if changes were made. 


$$
\begin{aligned}
& y^{(i)}(0)=y^{(j)}(1)=0, \quad 0 \leq i \leq k-1,0 \leq j \leq n-k-2, \\
& y^{(n-1)}(1)=\sum_{i=1}^{m} \alpha_{i} y^{(n-1)}\left(\xi_{i}\right)
\end{aligned}
$$

where $1 \leq k \leq n-1,0<\xi_{1}<\xi_{2}<\cdots<\xi_{m}<1$. Integral boundary value problems have also gained many people's attention and have been applied to many fields, such as physics, chemistry, and engineering, see [11, 13, 22, 29-31, 35]. Besides, the subject of fractional differential equations has attracted much attention, see $[1-5,7,10,12,15,23,24,27$, 28, 32-34, 36-39, 42]. For example, in [5], Zhang and Bai investigated the existence of solutions for the following $m$-point boundary value problems:

$$
\begin{aligned}
& D_{0^{+}}^{\alpha} u(t)=f\left(t, u(t), D_{0^{+}}^{\alpha-1} u(t)\right)+e(t), \quad t \in(0,1), \alpha \in(1,2], \\
& \left.I_{0^{+}}^{\alpha} u(t)\right|_{t=0}=0, \quad D_{0^{+}}^{\alpha-1} u(1)=\sum_{i=1}^{m-2} \beta_{i} D_{0^{+}}^{\alpha-1} u\left(\eta_{i}\right)
\end{aligned}
$$

by using the coincidence degree theory of Mawhin. Very recently, in [2], the authors considered boundary value problem (1) under the nonresonance condition $v_{1} v_{4}+v_{2} v_{3} \neq 0$. They established the existence and uniqueness results of BVP (1) by using the standard fixed point theorems.

Inspired by the work above, in this paper, we intend to discuss the boundary value problem (1) under the resonance condition $v_{1} v_{4}+v_{2} v_{3}=0$. We shall study resonant BVP (1) in three different cases of $\operatorname{dim} \operatorname{ker} L=1$. Different from the above results, the boundary conditions we study are nonlocal Erdélyi-Kober type integral and generalized RiemannLiouville type integral. To the best of our knowledge, it is innovative to study the boundary value problem with the nonlocal Erdélyi-Kober type integral and generalized RiemannLiouville type integral by using the method of Mawhin continuation theorem.

The organization of this paper is as follows. In Sect. 2, we provide some definitions, lemmas, and Mawhin continuation theorem which will be used to prove the main results. In Sect. 3, we will give our main results and the proof, some lemmas will also be given to prove the solvability of BVP (1).

\section{Preliminaries}

Firstly, for the convenience of the reader, we recall some definitions and lemmas.

Definition 2.1 $([2,18])$ The fractional integral of order $q$ with the lower limit zero for a function $f$ is defined by

$$
J^{q} f(t)=\frac{1}{\Gamma(q)} \int_{0}^{t} \frac{f(s)}{(t-s)^{1-q}} d s, \quad t>0, q>0,
$$

provided the right-hand side is point-wise defined on $[0, \infty), \Gamma(\cdot)$ is the gamma function.

Definition 2.2 ([2]) The generalized fractional integral of order $q>0$ and $\rho>0$ for a function $f(t)$ is defined by

$$
{ }^{\rho} I^{q} f(t)=\frac{\rho^{1-q}}{\Gamma(q)} \int_{0}^{t} \frac{s^{\rho-1} f(s)}{\left(t^{\rho}-s^{\rho}\right)^{1-q}} d s, \quad t \in(0, \infty),
$$

provided the right-hand side is point-wise defined on $(0, \infty)$. 
Definition 2.3 ([2]) The Erdélyi-Kober fractional integral of order $\delta>0$ with $\eta>0$ and $\gamma \in \mathbb{R}$ of a continuous function $f(t)$ is defined as

$$
I_{\eta}^{\gamma, \delta} f(t)=\frac{\eta t^{-\eta(\delta+\gamma)}}{\Gamma(\delta)} \int_{0}^{t} \frac{s^{\eta \gamma+\eta-1} f(s)}{\left(t^{\eta}-s^{\eta}\right)^{1-\delta}} d s, \quad t \in(0, \infty)
$$

provided the right-hand side is point-wise defined on $\mathbb{R}_{+}$.

Definition 2.4 ([2, 18]) The Riemann-Liouville fractional derivative of order $q>0$, $n-1<q<n, n \in \mathbb{N}$ can be written as

$$
D_{0^{+}}^{q} f(t)=\frac{1}{\Gamma(n-q)}\left(\frac{d}{d t}\right)^{n} \int_{0}^{t}(t-s)^{n-q-1} f(s) d s,
$$

where the function $f(t)$ has absolutely continuous derivative up to order $(n-1)$.

Definition 2.5 $([2,18])$ The Caputo derivative of order $q$ for a function $f:[0, \infty) \rightarrow \mathbb{R}$ is defined as

$$
{ }^{c} D^{q} f(t)=D_{0^{+}}^{q}\left(f(t)-\sum_{k=0}^{n-1} \frac{t^{k}}{k !} f^{(k)}(0)\right), \quad t>0, n-1<q<n .
$$

Lemma 2.1 ([18]) Given that $x \in C^{1}[0,1]$ with a fractional derivative of order $q(1<q<2)$ that belongs to $C(0,1) \cap L(0,1)$, then

$$
J^{q} D^{q} x(t)=x(t)-x(0)-x^{\prime}(0) t .
$$

Lemma 2.2 ([2]) Let $\delta, \eta>0, \gamma, q \in \mathbb{R}$, then we can get

$$
I_{\eta}^{\gamma, \delta} t^{q}=\frac{t^{q} \Gamma\left(\gamma+\frac{q}{\eta}+1\right)}{\Gamma\left(\gamma+\frac{q}{\eta}+\delta+1\right)}
$$

Lemma 2.3 ([2]) Let $q, p>0$, then we have

$$
{ }^{\rho} I^{q} t^{p}=\frac{\Gamma\left(\frac{p+\rho}{\rho}\right)}{\Gamma\left(\frac{p+\rho q+\rho}{\rho}\right)} \frac{t^{p+\rho q}}{\rho^{q}} .
$$

Definition 2.6 ([26]) Assume that $X$ and $Y$ are real Banach spaces, $L: \operatorname{dom} L \subset X \rightarrow Y$ is a Fredholm operator of index zero if the following conditions hold:

(1) $\operatorname{Im} L$ is a closed subspace of $Y$;

(2) $\operatorname{dim} \operatorname{Ker} L=\operatorname{codim} \operatorname{Im} L<\infty$.

Let $X, Y$ be real Banach spaces, $L: \operatorname{dom} L \subset X \rightarrow Y$ be a Fredholm operator of index zero, and $N: X \rightarrow Y$ be a nonlinear continuous map. $P: X \rightarrow X, Q: Y \rightarrow Y$ are continuous projectors such that

$$
\operatorname{Im} P=\operatorname{ker} L, \quad \operatorname{ker} Q=\operatorname{Im} L, \quad X=\operatorname{ker} L \oplus \operatorname{ker} P, \quad Y=\operatorname{Im} L \oplus \operatorname{Im} Q .
$$


It follows that

$$
\left.L\right|_{\operatorname{dom} L \cap \operatorname{Ker} P}: \operatorname{dom} L \cap \operatorname{ker} P \rightarrow \operatorname{Im} L
$$

is invertible, and the inverse of the mapping is denoted by $K_{P}$ (generalized inverse operator of $L$ ). Let $\Omega$ be an open bounded subset of $X$ with $\operatorname{dom} L \cap \Omega \neq \emptyset$, the mapping $N: X \rightarrow Y$ will be called $L$-compact on $\bar{\Omega}$ if $Q N(\bar{\Omega})$ is bounded and $K_{P}(I-Q) N: \bar{\Omega} \rightarrow X$ is compact.

Theorem 2.1 (Mawhin continuation theorem [26]) Let $L: \operatorname{dom} L \subset X \rightarrow Y$ be a Fredholm operator of index zero and $N$ be L-compact on $\bar{\Omega}$. The equation $L \varphi=N \varphi$ has at least one solution in $\operatorname{dom} L \cap \bar{\Omega}$ if the following conditions are satisfied:

(1) $L x \neq \lambda N x$ for every $(x, \lambda) \in[(\operatorname{dom} L \backslash \operatorname{ker} L) \cap \partial \Omega] \times(0,1)$;

(2) $N x \notin \operatorname{Im} L$ for every $x \in \operatorname{ker} L \cap \partial \Omega$;

(3) $\operatorname{deg}\left(\left.Q N\right|_{\operatorname{ker} L}, \Omega \cap \operatorname{ker} L, 0\right) \neq 0$, where $Q: Y \rightarrow Y$ is a projection such that $\operatorname{Im} L=\operatorname{ker} Q$.

Let $Y=C[0, T]$ with the norm $\|x\|_{\infty}=\max _{t \in[0,1]}|x(t)|$ and $X=C^{1}[0, T]$ with the norm $\|x\|=\max \left\{\|x\|_{\infty},\left\|x^{\prime}\right\|_{\infty}\right\}$. Obviously, $X$ and $Y$ are Banach spaces.

An operator $L$ is defined as $L: L x(t)={ }^{c} D^{q} x(t)$ with

$$
\operatorname{dom} L=\left\{x \in X:{ }^{c} D^{q} x \in Y, x(0)=\alpha I_{\eta}^{\gamma, \delta} x(\zeta), x(T)=\beta^{\rho} I^{p} x(\xi)\right\}
$$

Define the operator $N: X \rightarrow Y$ as follows:

$$
(N x)(t)=f\left(t, x(t), x^{\prime}(t)\right)
$$

So problem (1) becomes $L x=N x$.

Let

$$
\begin{array}{ll}
v_{1}=1-\alpha \frac{\Gamma(\gamma+1)}{\Gamma(\gamma+\delta+1)}, & v_{2}=\alpha \zeta \frac{\Gamma\left(\gamma+\frac{1}{\eta}+1\right)}{\Gamma\left(\gamma+\frac{1}{\eta}+\delta+1\right)} \\
v_{3}=1-\beta \frac{\xi^{\rho q}}{\rho^{q}} \frac{1}{\Gamma(q+1)}, & v_{4}=T-\beta \frac{\xi^{\rho q+1}}{\rho^{q}} \frac{\Gamma\left(\frac{1+\rho}{\rho}\right)}{\Gamma\left(\frac{1+\rho q+\rho}{\rho}\right)},
\end{array}
$$

then we consider the following three resonant conditions:

(A1) $v_{1}=v_{3}=0, v_{2} \neq 0, v_{4} \neq 0$;

(A2) $v_{2}=v_{4}=0, v_{1} \neq 0, v_{3} \neq 0$;

(A3) $v_{i} \neq 0(i=1,2,3,4), v_{1} v_{4}+v_{2} v_{3}=0$.

Lemma 2.4 Assume that (A1) holds. Then there exists $z \in Y$ such that

$$
v_{2}\left(\beta^{\rho} I^{p} J^{q} z(\xi)-J^{q} z(T)\right)+\alpha v_{4} I_{\eta}^{\gamma, \delta} J^{q} z(\zeta)=1 .
$$

Proof We define two linear functionals $B_{1}, B_{2}: X \rightarrow \mathbb{R}$ as follows:

$$
\begin{aligned}
& B_{1} x=x(0)-\alpha I_{\eta}^{\gamma, \delta} x(\zeta), \quad x \in X, \\
& B_{2} x=x(T)-\beta^{\rho} I^{p} x(\xi), \quad x \in X .
\end{aligned}
$$


Let $\varphi(t)=1, \psi(t)=t$. It follows from $(A 1)$ and Lemmas 2.2 and 2.3 that

$$
\begin{array}{ll}
B_{1} \varphi=\varphi(0)-\alpha I_{\eta}^{\gamma, \delta} \varphi(\zeta)=v_{1}=0, & B_{1} \psi=\psi(0)-\alpha I_{\eta}^{\gamma, \delta} \psi(\zeta)=-v_{2}, \\
B_{2} \varphi=\varphi(T)-\beta^{\rho} I^{p} \varphi(\xi)=v_{3}=0, & B_{2} \psi=\psi(T)-\beta^{\rho} I^{p} \psi(\xi)=v_{4} .
\end{array}
$$

So, (2) can be rewritten by

$$
B_{1} \psi \cdot B_{2}\left(J^{q} z\right)-B_{2} \psi \cdot B_{1}\left(J^{q} z\right)=1
$$

For convenience, set

$$
B x=B_{1} \psi \cdot B_{2}(x)-B_{2} \psi \cdot B_{1}(x)
$$

If there is $\widetilde{z} \in Y$ such that $B \widetilde{z} \neq 0$ and, as a result, $z=\frac{\widetilde{z}}{B \widetilde{z}} \in Y$ with $B z=1$. Assume the contrary. Then $B\left(J^{q} z\right)=0$ for all $z \in Y$, and, in particular, for integer $n \geq 2$,

$$
\frac{\Gamma(n+1)}{\Gamma(n-q+1)} B\left(J^{q} t^{n-q}\right)=B\left(t^{n}\right)=0 .
$$

By (3), $B(1)=B(t)=0$. Therefore, $B(g)=0$ for every polynomial $g$. Note that $B \neq 0$ on all of $X$, there exists $x_{0} \in X$ such that $B x_{0} \neq 0$. Thus, there exists a sequence of polynomials $g_{n}$ such that $\left\|x_{0}-g_{n}\right\|_{X}<\frac{1}{n}$. So, we deduce that

$$
0 \neq\left|B x_{0}\right|=\left|B\left(x_{0}-g_{n}\right)+B g_{n}\right|=\left|B\left(x_{0}-g_{n}\right)\right| \leq\|B\|\left\|x_{0}-g_{n}\right\|_{X}<\frac{\|B\|}{n}
$$

for all integer $n$, which is a contradiction. Thus, there exists $z \in Y$ satisfying (2). Thus the lemma holds.

Similar to the proof of Lemma 2.4, we also get the following lemmas.

Lemma 2.5 Assume that (A2) holds. Then there exists $z_{1} \in Y$ such that

$$
J^{q} z_{1}(T)-\beta^{\rho} I^{p} J^{q} z_{1}(\xi)=1
$$

Lemma 2.6 Assume that (A3) holds. Then there exists $z_{2} \in Y$ such that

$$
v_{2}\left(\beta^{\rho} I^{p} J^{q} z_{2}(\xi)-J^{q} z_{2}(T)\right)+\alpha v_{4} I_{\eta}^{\gamma, \delta} J^{q} z_{2}(\zeta)=1 .
$$

Remark 2.1 The main idea of Lemmas 2.4, 2.5, and 2.6 comes from $[16,19,20]$.

\section{Main results}

Assume that the following conditions hold in this paper:

(H1) $f:[0,1] \times \mathbb{R}^{2} \rightarrow \mathbb{R}$ is a continuous function.

(H2) There exist nonnegative functions $u, v, w \in C[0, T]$ such that

$$
\left|f\left(t, x_{1}, x_{2}\right)\right| \leq u(t)\left|x_{1}\right|+v(t)\left|x_{2}\right|+w(t), \quad t \in[0, T], x_{1}, x_{2} \in \mathbb{R} .
$$


(H3) There exists a constant $M>0$ such that if $|x(t)|+\left|x^{\prime}(t)\right|>M$ for all $t \in[0, T]$, then

$$
v_{2}\left(\beta^{\rho} I^{p} J^{q} N x(\xi)-J^{q} N x(T)\right)+\alpha v_{4} I_{\eta}^{\gamma, \delta} J^{q} N x(\zeta) \neq 0 .
$$

(H3') There exists a constant $M>0$ such that if $\left|x^{\prime}(t)\right|>M$ for all $t \in[0, T]$, then

$$
\beta^{\rho} I^{p} J^{q} N x(\xi)-J^{q} N x(T) \neq 0 .
$$

(H4) There is a constant $D>0$ such that either

$$
c v_{2}\left(\beta^{\rho} I^{p} J^{q} N \phi_{1}(\xi)-J^{q} N \phi_{1}(T)\right)+c \alpha v_{4} I_{\eta}^{\gamma, \delta} J^{q} N \phi_{1}(\zeta)>0
$$

or

$$
c v_{2}\left(\beta^{\rho} I^{p} J^{q} N \phi_{1}(\xi)-J^{q} N \phi_{1}(T)\right)+c \alpha v_{4} I_{\eta}^{\gamma, \delta} J^{q} N \phi_{1}(\zeta)<0
$$

holds if $|c|>D$, where $\phi_{1}(t)=c$.

$\left(\mathrm{H} 4^{\prime}\right)$ There is a constant $D>0$ such that either

$$
c \beta^{\rho} I^{p} J^{q} N \phi_{2}(\xi)-c J^{q} N \phi_{2}(T)>0
$$

or

$$
c \beta^{\rho} I^{p} J^{q} N \phi_{2}(\xi)-c J^{q} N \phi_{2}(T)<0
$$

holds if $|c|>D$, where $\phi_{2}(t)=c t$.

$\left(\mathrm{H} 4^{\prime \prime}\right)$ There is a constant $D>0$ such that either

$$
c v_{2}\left(\beta^{\rho} I^{p} J^{q} N \phi_{3}(\xi)-J^{q} N \phi_{3}(T)\right)+c \alpha v_{4} I_{\eta}^{\gamma, \delta} J^{q} N \phi_{3}(\zeta)>0
$$

or

$$
c v_{2}\left(\beta^{\rho} I^{p} J^{q} N \phi_{3}(\xi)-J^{q} N \phi_{3}(T)\right)+c \alpha v_{4} I_{\eta}^{\gamma, \delta} J^{q} N \phi_{3}(\zeta)<0
$$

holds if $|c|>D$, where $\phi_{3}(t)=c(1+k t), k=\frac{v_{1}}{v_{2}}$.

Then we can present the following theorem.

Theorem 3.1 Suppose that $(A 1)$ and $(\mathrm{H} 1)-(\mathrm{H} 4)$ are satisfied, then there must be at least one solution of problem (1) in $X$ provided that $2 T^{q}\|u\|_{\infty}+2 T^{q-1}\|v\|_{\infty}<\Gamma(q)$.

To prove the theorem, we need the following lemmas.

Lemma 3.1 Assume that (A1) holds, then $L: \operatorname{dom} L \subset X \rightarrow Y$ is a Fredholm operator with index zero. And a linear continuous projector $P: X \rightarrow X$ can be defined by

$$
(P x)(t)=x(0) .
$$


Furthermore, define the linear operator $K_{p}: \operatorname{Im} L \rightarrow \operatorname{dom} L \cap \operatorname{ker} P$ as follows:

$$
\left(K_{p} y\right)(t)=\frac{1}{\Gamma(q)} \int_{0}^{t}(t-s)^{q-1} y(s) d s-\frac{\alpha I_{\eta}^{\gamma, \delta} J^{q} y(\zeta)}{v_{2}} \cdot t
$$

such that $K_{p}=\left(\left.L\right|_{\operatorname{dom} L \cap \operatorname{ker} P}\right)^{-1}$.

Proof Let $\varphi(t)=1, \psi(t)=t$. From $(A 1)$ and Lemma 2.4, we can easily get

$\operatorname{ker} L=\{c, c \in \mathbb{R}\}$.

Moreover, we can obtain that

$$
\operatorname{Im} L=\left\{y \in Y: v_{2}\left(\beta^{\rho} I^{p} J^{q} y(\xi)-J^{q} y(T)\right)+\alpha v_{4} I_{\eta}^{\gamma, \delta} J^{q} y(\zeta)=0\right\}
$$

On the one hand, suppose $y \in \operatorname{Im} L$, then there exists $x \in \operatorname{dom} L$ such that

$$
y=L x \in Y .
$$

Then we have

$$
x(t)=J^{q} y(t)+c_{0}+c_{1} t=\frac{1}{\Gamma(q)} \int_{0}^{t}(t-s)^{q-1} y(s) d s+c_{0}+c_{1} t,
$$

where $c_{0}, c_{1} \in \mathbb{R}$. Furthermore, for $x \in \operatorname{dom} L$,

$$
\begin{aligned}
x(0) & =\alpha I_{\eta}^{\gamma, \delta} x(\zeta)=\alpha I_{\eta}^{\gamma, \delta} J^{q} y(\zeta)+c_{0} \alpha I_{\eta}^{\gamma, \delta} \varphi(\zeta)+c_{1} \alpha I_{\eta}^{\gamma, \delta} \psi(\zeta) \\
& =\alpha I_{\eta}^{\gamma, \delta} J^{q} y(\zeta)+c_{0}+c_{1} v_{2},
\end{aligned}
$$

and

$$
x(0)=J^{q} y(t)+c_{0}+\left.c_{1} t\right|_{t=0}=c_{0} .
$$

The above two equalities imply that

$$
\alpha I_{\eta}^{\gamma, \delta} J^{q} y(\zeta)+c_{1} \nu_{2}=0 .
$$

Using (3) and (7), we get the system

$$
\begin{aligned}
x(T) & =\beta^{\rho} I^{p} x(\xi)=\beta^{\rho} I^{p} J^{q} y(\xi)+c_{0} \beta^{\rho} I^{p} \varphi(\xi)+c_{1} \beta^{\rho} I^{p} \psi(\xi) \\
& =\beta^{\rho} I^{p} J^{q} y(\xi)+c_{0}+c_{1}\left(T-v_{4}\right), \\
x(T) & =J^{q} y(t)+c_{0}+\left.c_{1} t\right|_{t=T}=J^{q} y(T)+c_{0}+c_{1} T .
\end{aligned}
$$

From this together with the second boundary value condition of (1), we can get

$$
\beta^{\rho} I^{p} J^{q} y(\xi)-J^{q} y(T)=c_{1} v_{4} .
$$


By using the eliminated element method, equalities (8) and (9) are changed into the equality

$$
\nu_{2}\left(\beta^{\rho} I^{p} J^{q} y(\xi)-J^{q} y(T)\right)+\alpha v_{4} I_{\eta}^{\gamma, \delta} J^{q} y(\zeta)=0
$$

So we obtain that

$$
\operatorname{Im} L \subset\left\{y \in Y: v_{2}\left(\beta^{\rho} I^{p} J^{q} y(\xi)-J^{q} y(T)\right)+\alpha v_{4} I_{\eta}^{\gamma, \delta} J^{q} y(\zeta)=0\right\}
$$

On the other hand, if $y \in Y$ satisfies $v_{2}\left(\beta^{\rho} I^{p} J^{q} y(\xi)-J^{q} y(T)\right)+\alpha v_{4} I_{\eta}^{\gamma, \delta} J^{q} y(\zeta)=0$, we let

$$
x(t)=J^{q} y(t)-\frac{\alpha I_{\eta}^{\gamma, \delta} J^{q} y(\zeta)}{v_{2}} \cdot t .
$$

Then we conclude that

$$
L x(t)={ }^{c} D^{q} x(t)=y(t)
$$

and

$$
\begin{aligned}
& x(0)=0, \\
& \begin{aligned}
\alpha I_{\eta}^{\gamma, \delta} x(\zeta) & =\alpha I_{\eta}^{\gamma, \delta} J^{q} y(\zeta)-\frac{\alpha I_{\eta}^{\gamma, \delta} J^{q} y(\zeta)}{v_{2}} \cdot \alpha I_{\eta}^{\gamma, \delta} \psi(\zeta) \\
& =\alpha I_{\eta}^{\gamma, \delta} J^{q} y(\zeta)-\frac{\alpha I_{\eta}^{\gamma, \delta} J^{q} y(\zeta)}{v_{2}} \cdot v_{2}=0 .
\end{aligned}
\end{aligned}
$$

Besides,

$$
\begin{aligned}
x(T)=J^{q} y(T)-\frac{\alpha I_{\eta}^{\gamma, \delta} J^{q} y(\zeta)}{v_{2}} \cdot T, & \\
\beta^{\rho} I^{p} x(\xi) & =\beta^{\rho} I^{p} J^{q} y(\xi)-\frac{\alpha I_{\eta}^{\gamma, \delta} J^{q} y(\zeta)}{v_{2}} \cdot \beta^{\rho} I^{p} \psi(\xi) \\
& =\beta^{\rho} I^{p} J^{q} y(\xi)-\frac{\alpha I_{\eta}^{\gamma, \delta} J^{q} y(\zeta)}{v_{2}} \cdot\left(T-v_{4}\right) \\
& =-\frac{\alpha v_{4} I_{\eta}^{\gamma, \delta} J^{q} y(\zeta)}{v_{2}}+J^{q} y(T)-\frac{\alpha I_{\eta}^{\gamma, \delta} J^{q} y(\zeta)}{v_{2}} \cdot\left(T-v_{4}\right) \\
& =J^{q} y(T)-\frac{\alpha I_{\eta}^{\gamma, \delta} J^{q} y(\zeta)}{v_{2}} \cdot T,
\end{aligned}
$$

therefore

$$
x(0)=\alpha I_{\eta}^{\gamma, \delta} x(\zeta), \quad x(T)=\beta^{\rho} I^{p} x(\xi)
$$

That is, $x \in \operatorname{dom} L$, then $y \in \operatorname{Im} L$. In conclusion,

$$
\operatorname{Im} L=\left\{y \in Y: \nu_{2}\left(\beta^{\rho} I^{p} J^{q} y(\xi)-J^{q} y(T)\right)+\alpha v_{4} I_{\eta}^{\gamma, \delta} J^{q} y(\zeta)=0\right\} .
$$


We define the linear operator $P: X \rightarrow X$ as

$$
(P x)(t)=x(0) .
$$

It is obvious that $P^{2} x=P x$ and $\operatorname{Im} P=\operatorname{ker} L$. For any $x \in X$, together with $x=(x-P x)+P x$, we have $X=\operatorname{ker} P+\operatorname{ker} L$. It is easy to obtain that $\operatorname{ker} L \cap \operatorname{ker} P=\emptyset$, which implies

$$
X=\operatorname{ker} P \oplus \operatorname{ker} L .
$$

Next the operator $Q: Y \rightarrow Y$ is defined as follows:

$$
\begin{aligned}
(Q y)(t) & =\left(v_{2}\left(\beta^{\rho} I^{p} J^{q} y(\xi)-J^{q} y(T)\right)+\alpha v_{4} I_{\eta}^{\gamma, \delta} J^{q} y(\zeta)\right) z(t) \\
& =B\left(J^{q} y\right) z(t),
\end{aligned}
$$

where $B$ is given by (4) and $z \in Y$ satisfying $B\left(J^{q} z\right)=1$.

Obviously, $Q$ is a projection operator such that $\operatorname{ker} Q=\operatorname{Im} L$ and $\operatorname{Im} L=\{c z(t): c \in \mathbb{R}\}$. For any $y \in Y$, because $y=(y-Q y)+Q y$, we have $Y=\operatorname{Im} L+\operatorname{Im} Q$. Moreover, by a simple calculation, we can get $\operatorname{Im} Q \cap \operatorname{Im} L=\emptyset$. Above all, $Y=\operatorname{Im} L \oplus \operatorname{Im} Q$.

To sum up, we can get that $\operatorname{Im} L$ is a closed subspace of $Y$; $\operatorname{dim} \operatorname{ker} L=\operatorname{codim} \operatorname{Im} L<+\infty$; that is, $L$ is a Fredholm operator of index zero.

We now define the operator $K_{p} y: Y \rightarrow X$ as follows:

$$
\begin{aligned}
\left(K_{p} y\right)(t) & =J^{q} y(t)-\frac{\alpha I_{\eta}^{\gamma, \delta} J^{q} y(\zeta)}{v_{2}} \cdot t \\
& =\frac{1}{\Gamma(q)} \int_{0}^{t}(t-s)^{q-1} y(s) d s-\frac{\alpha I_{\eta}^{\gamma, \delta} J^{q} y(\zeta)}{\nu_{2}} \cdot t
\end{aligned}
$$

For any $y \in \operatorname{Im} L$, we have

$$
\left(K_{p} y\right)(0)=\alpha I_{\eta}^{\gamma, \delta}\left(K_{p} y\right)(\zeta), \quad\left(K_{p} y\right)(T)=\beta^{\rho} I^{p}\left(K_{p} y\right)(\xi),
$$

then $\left(K_{p} y\right)(t) \in \operatorname{dom} L$. In addition, $\left(K_{p} y\right)(0)=0$, which means $K_{p} y \in \operatorname{ker} P$. Therefore

$$
K_{p} y \in \operatorname{dom} L \cap \operatorname{ker} P, \quad y \in \operatorname{Im} L .
$$

Next we will prove that $K_{p}$ is the inverse of $\left.L\right|_{\operatorname{dom} L \cap \operatorname{ker} P}$. It is clear that

$$
\left(L K_{p} y\right)(t)=y(t), \quad y \in \operatorname{Im} L
$$

By Lemma 2.1, for each $x \in \operatorname{dom} L \cap \operatorname{ker} P$, we have $x(0)=0$ and

$$
\begin{aligned}
\left(K_{p} L x\right)(t) & =J^{q} D^{q} x(t)-\frac{\alpha I_{\eta}^{\gamma, \delta} J^{q} D^{q} x(\zeta)}{v_{2}} \cdot t \\
& =x(t)-x(0)-x^{\prime}(0) t-\frac{\alpha I_{\eta}^{\gamma, \delta} x(\zeta)-x(0) \alpha I_{\eta}^{\gamma, \delta} \varphi(\zeta)-x^{\prime}(0) \alpha I_{\eta}^{\gamma, \delta} \psi(\zeta)}{v_{2}} \cdot t \\
& =x(t)-x(0)-x^{\prime}(0) t-\frac{x(0)-x(0) \alpha I_{\eta}^{\gamma, \delta} \varphi(\zeta)-x^{\prime}(0) \alpha I_{\eta}^{\gamma, \delta} \psi(\zeta)}{v_{2}} \cdot t
\end{aligned}
$$




$$
\begin{aligned}
& =x(t)-x^{\prime}(0) t+x^{\prime}(0) t \\
& =x(t) .
\end{aligned}
$$

This implies that $K_{p} L x=x$. So $K_{p}=\left(\left.L\right|_{\operatorname{dom} L \cap \operatorname{ker} P}\right)^{-1}$. Thus the lemma holds.

Lemma 3.2 $N$ is L-compact on $\bar{\Omega}$ if $\operatorname{dom} L \cap \bar{\Omega} \neq \emptyset$, where $\Omega$ is a bounded open subset of $X$.

Proof It follows from the continuity of $f$ in condition (H1) and $z \in Y$ that $(I-Q) N(\bar{\Omega})$ is bounded. In addition,

$$
\left\{\frac{1}{\Gamma(q)} \int_{0}^{t}(t-s)^{q-1} y(s) d s-\frac{\alpha I_{\eta}^{\gamma, \delta} J^{q} y(\zeta)}{v_{2}} \cdot t: y \in(I-Q) N(\bar{\Omega})\right\}
$$

and

$$
\left\{\frac{1}{\Gamma(q-1)} \int_{0}^{t}(t-s)^{q-2} y(s) d s-\frac{\alpha I_{\eta}^{\gamma, \delta} J^{q} y(\zeta)}{v_{2}}: y \in(I-Q) N(\bar{\Omega})\right\}
$$

are equi-continuous and uniformly bounded. By Ascoli-Arzela theorem, we get $K_{p}(I-Q) N: \bar{\Omega} \rightarrow X$ is compact. Thus, $N$ is $L$-compact. The proof is completed.

Lemma 3.3 The set $\Omega_{1}=\{x \in \operatorname{dom} L \backslash \operatorname{ker} L: L x=\lambda N x, \lambda \in[0,1]\}$ is bounded if (H1)-(H3) are satisfied.

Proof Take $x \in \Omega_{1}$, then $x \notin \operatorname{ker} L$, so $\lambda \neq 0$ and $N x \in \operatorname{Im} L$. Thus we have

$$
\left(v_{2}\left(\beta^{\rho} I^{p} J^{q} N x(\xi)-J^{q} N x(T)\right)+\alpha v_{4} I_{\eta}^{\gamma, \delta} J^{q} N x(\zeta)\right) z(t)=0,
$$

where $z \in Y$ satisfying $B\left(J^{q} z\right)=1$. So we get

$$
v_{2}\left(\beta^{\rho} I^{p} J^{q} N x(\xi)-J^{q} N x(T)\right)+\alpha v_{4} I_{\eta}^{\gamma, \delta} J^{q} N x(\zeta)=0 .
$$

According to (H3), there exists at least a point $t_{0} \in[0, T]$ such that

$$
\left|x\left(t_{0}\right)\right|+\left|x^{\prime}\left(t_{0}\right)\right| \leq M
$$

Using the Newton-Leibnitz formula, we have

$$
\|x\|_{\infty}=\max _{t \in[0, T]}|x(t)|=\max _{t \in[0, T]}\left|x\left(t_{0}\right)+\int_{t_{0}}^{t} x^{\prime}(s) d s\right| \leq M+T\left\|x^{\prime}\right\|_{\infty} .
$$

In addition, for $L x=\lambda N x$ and $x \in \operatorname{dom} L$, we have

$$
x(t)=\frac{\lambda}{\Gamma(q)} \int_{0}^{t}(t-s)^{q-1} f\left(s, x(s), x^{\prime}(s)\right) d s+x(0)+x^{\prime}(0) t
$$

and

$$
x^{\prime}(t)=\frac{\lambda}{\Gamma(q-1)} \int_{0}^{t}(t-s)^{q-2} f\left(s, x(s), x^{\prime}(s)\right) d s+x^{\prime}(0) .
$$


Take $t=t_{0}$ in (12), we get

$$
x^{\prime}\left(t_{0}\right)=\frac{\lambda}{\Gamma(q-1)} \int_{0}^{t_{0}}\left(t_{0}-s\right)^{q-2} f\left(s, x(s), x^{\prime}(s)\right) d s+x^{\prime}(0) .
$$

This together with $\left|x^{\prime}\left(t_{0}\right)\right| \leq M$ and (11) implies that

$$
\begin{aligned}
\left|x^{\prime}(0)\right| & \leq\left|x^{\prime}\left(t_{0}\right)\right|+\frac{\lambda}{\Gamma(q-1)} \int_{0}^{t_{0}}\left(t_{0}-s\right)^{q-2}\left|f\left(s, x(s), x^{\prime}(s)\right)\right| d s \\
& \leq M+\frac{\lambda}{\Gamma(q-1)} \int_{0}^{t_{0}}\left(t_{0}-s\right)^{q-2}\left[u(s)|x(s)|+v(s)\left|x^{\prime}(s)\right|+w(s)\right] d s \\
& \leq M+\frac{T^{q-1}}{\Gamma(q)}\left(\|u\|_{\infty}\|x\|_{\infty}+\|v\|_{\infty}\left\|x^{\prime}\right\|_{\infty}+\|w\|_{\infty}\right) \\
& \leq M+\frac{T^{q-1}}{\Gamma(q)}\left(\|u\|_{\infty}\left(M+T\left\|x^{\prime}\right\|_{\infty}\right)+\|v\|_{\infty}\left\|x^{\prime}\right\|_{\infty}+\|w\|_{\infty}\right) \\
& =M+\frac{T^{q-1}}{\Gamma(q)}\left(M\|u\|_{\infty}+\|w\|_{\infty}\right)+\frac{T^{q}\|u\|_{\infty}+T^{q-1}\|v\|_{\infty}}{\Gamma(q)}\left\|x^{\prime}\right\|_{\infty}
\end{aligned}
$$

Then we conclude that

$$
\begin{aligned}
\left|x^{\prime}(t)\right| \leq & \frac{\lambda}{\Gamma(q-1)} \int_{0}^{t}(t-s)^{q-2}\left|f\left(s, x(s), x^{\prime}(s)\right)\right| d s+\left|x^{\prime}(0)\right| \\
\leq & \frac{T^{q-1}}{\Gamma(q)}\left(\|u\|_{\infty}\|x\|_{\infty}+\|v\|_{\infty}\left\|x^{\prime}\right\|_{\infty}+\|w\|_{\infty}\right) \\
& +M+\frac{T^{q-1}}{\Gamma(q)}\left(M\|u\|_{\infty}+\|w\|_{\infty}\right)+\frac{T^{q}\|u\|_{\infty}+T^{q-1}\|v\|_{\infty}}{\Gamma(q)}\left\|x^{\prime}\right\|_{\infty} \\
\leq & \frac{T^{q-1}}{\Gamma(q)}\left(\|u\|_{\infty}\left(M+T\left\|x^{\prime}\right\|_{\infty}\right)+\|v\|_{\infty}\left\|x^{\prime}\right\|_{\infty}+\|w\|_{\infty}\right) \\
& +M+\frac{T^{q-1}}{\Gamma(q)}\left(M\|u\|_{\infty}+\|w\|_{\infty}\right)+\frac{T^{q}\|u\|_{\infty}+T^{q-1}\|v\|_{\infty}}{\Gamma(q)}\left\|x^{\prime}\right\|_{\infty} \\
= & M+2 \frac{T^{q-1}}{\Gamma(q)}\left(M\|u\|_{\infty}+\|w\|_{\infty}\right)+2 \frac{T^{q}\|u\|_{\infty}+T^{q-1}\|v\|_{\infty}}{\Gamma(q)}\left\|x^{\prime}\right\|_{\infty} .
\end{aligned}
$$

Therefore, we can obtain that

$$
\left\|x^{\prime}\right\|_{\infty} \leq \frac{M \Gamma(q)+2 M T^{q-1}\|u\|_{\infty}+2 T^{q-1}\|w\|_{\infty}}{\Gamma(q)-2 T^{q}\|u\|_{\infty}-2 T^{q-1}\|v\|_{\infty}}=M_{1}
$$

Combining this with (11), we have

$$
\|x\|_{\infty} \leq M+T\left\|x^{\prime}\right\|_{\infty} \leq M+T M_{1}
$$

Then $\Omega_{1}$ is bounded. The proof of the lemma is completed.

Lemma 3.4 The set $\Omega_{2}=\{x: x \in \operatorname{ker} L, N x \in \operatorname{Im} L\}$ is bounded if (H1), (H4) hold. 
Proof Let $x \in \Omega_{2}$, then $x(t) \equiv c$ and $N x \in \operatorname{Im} L$, so we can get

$$
v_{2}\left(\beta^{\rho} I^{p} J^{q} N x(\xi)-J^{q} N x(T)\right)+\alpha v_{4} I_{\eta}^{\gamma, \delta} J^{q} N x(\zeta)=0 .
$$

According to (H4), we have $|c| \leq D$, that is to say, $\Omega_{2}$ is bounded. We complete the proof

Lemma 3.5 The set $\Omega_{3}=\{x \in \operatorname{ker} L: \lambda x+\alpha(1-\lambda) J Q N x=0, \lambda \in[0,1]\}$ is bounded if conditions (H1), (H4) are satisfied, where $J: \operatorname{Im} Q \rightarrow \operatorname{ker} L$ is a linear isomorphism defined by

$$
J\left(c z_{1}\right)=c, \quad c \in \mathbb{R}
$$

and

$$
\alpha= \begin{cases}-1, & \text { if }(5) \text { holds } \\ 1, & \text { if }(6) \text { holds }\end{cases}
$$

where $z_{1}$ is introduced in Lemma 2.4.

Proof Suppose that $x \in \Omega_{3}$, we have $x(t)=c$ and

$$
\lambda x+\alpha(1-\lambda) J Q N x=0,
$$

thus we have

$$
\lambda c=-\alpha(1-\lambda)\left(v_{2}\left(\beta^{\rho} I^{p} J^{q} N x(\xi)-J^{q} N x(T)\right)+\alpha v_{4} I_{\eta}^{\gamma, \delta} J^{q} N x(\zeta)\right) .
$$

If $\lambda=0$, by condition (H4) we have $|c| \leq D$. If $\lambda=1$, then $c=0$. If $\lambda \in(0,1)$, we suppose $|c|>D$, then

$$
\lambda c^{2}=-\alpha(1-\lambda) c\left(v_{2}\left(\beta^{\rho} I^{p} J^{q} N x(\xi)-J^{q} N x(T)\right)+\alpha v_{4} I_{\eta}^{\gamma, \delta} J^{q} N x(\zeta)\right)<0
$$

which contradicts with $\lambda c^{2}>0$, so $|c| \leq D$. Then the lemma holds.

Theorem 3.1 can be proved now.

Proof of Theorem 3.1 Suppose that $\Omega \supset \bigcup_{i=1}^{3} \overline{\Omega_{i}} \cup\{0\}$ is a bounded open subset of $X$, from Lemma 3.2 we know that $N$ is $L$-compact on $\bar{\Omega}$. In view of Lemmas 3.3 and 3.4, we can get:

(i) $L x \neq \lambda N x$ for every $(x, \lambda) \in[(\operatorname{dom} L \backslash \operatorname{ker} L) \cap \partial \Omega] \times(0,1)$;

(ii) $N x \notin \operatorname{Im} L$ for every $x \in \operatorname{ker} L \cap \partial \Omega$.

Set $H(x, \lambda)=\lambda J x+\alpha(1-\lambda) Q N x$. It follows from Lemma 3.5 that we have $H(x, \lambda) \neq 0$ for any $x \in \partial \Omega \cap \operatorname{ker} L$. So, by the homotopic property of degree, we have

$$
\operatorname{deg}\left(\left.J Q N\right|_{\operatorname{ker} L}, \Omega \cap \operatorname{ker} L, 0\right)=\operatorname{deg}(\alpha I, \Omega \cap \operatorname{ker} L, 0) \neq 0 .
$$

All the conditions of Theorem 2.1 are satisfied. So there must be at least one solution of problem (1) in $X$. The proof of Theorem 3.1 is completed. 
Theorem 3.2 Suppose that $(A 2)$ and $(\mathrm{H} 1),(\mathrm{H} 2),\left(\mathrm{H}^{\prime}\right),\left(\mathrm{H}^{\prime}\right)$ are satisfied, then there must be at least one solution of problem (1) in $X$ provided that $2 T^{q}\|u\|_{\infty}+2 T^{q-1}\|v\|_{\infty}<\Gamma(q)$.

To prove the theorem, we need the following lemmas.

Lemma 3.6 Assume that (A2) holds, then $L: \operatorname{dom} L \subset X \rightarrow Y$ is a Fredholm operator with index zero. And the linear continuous projector $P: X \rightarrow X$ can be defined by

$$
(P x)(t)=\frac{x(T)}{T} t .
$$

Furthermore, define the linear operator $K_{p}: \operatorname{Im} L \rightarrow \operatorname{dom} L \cap \operatorname{ker} P$ as follows:

$$
\left(K_{p} y\right)(t)=\frac{1}{\Gamma(q)} \int_{0}^{t}(t-s)^{q-1} y(s) d s
$$

such that $K_{p}=\left(\left.L\right|_{\operatorname{dom} L \cap \mathrm{ker} P}\right)^{-1}$.

Proof Let $\varphi(t)=1, \psi(t)=t$. In view of $(A 2)$ we know

$$
\begin{aligned}
& \alpha=0, \\
& \varphi(0)-\alpha I_{\eta}^{\gamma, \delta} \varphi(\zeta)=1, \quad \psi(0)-\alpha I_{\eta}^{\gamma, \delta} \psi(\zeta)=0, \\
& \varphi(T)-\beta^{\rho} I^{p} \varphi(\xi)=v_{3}, \quad \psi(T)-\beta^{\rho} I^{p} \psi(\xi)=0,
\end{aligned}
$$

and we get

$$
\operatorname{ker} L=\{c t, c \in \mathbb{R}\}
$$

and

$$
\operatorname{Im} L=\left\{y \in Y: J^{q} y(T)=\beta^{\rho} I^{p} J^{q} y(\xi)\right\} .
$$

Besides, operators $P: X \rightarrow X, Q: Y \rightarrow Y$ can be defined as follows:

$$
(P x)(t)=\frac{x(T)}{T} t
$$

and

$$
(Q y)(t)=\left(J^{q} y(T)-\beta^{\rho} I^{p} J^{q} y(\xi)\right) z_{1}(t)
$$

where $z_{1} \in Y$ satisfying $J^{q} z_{1}(T)-\beta^{\rho} I^{p} J^{q} z_{1}(\xi)=1$. In addition, for each $x \in \operatorname{dom} L \cap \operatorname{ker} P$, we have $x(0)=0, x(T)=0$, then we get the generalized inverse operator of $L$ as follows:

$$
\left(K_{p} y\right)(t)=J^{q} y(t)=\frac{1}{\Gamma(q)} \int_{0}^{t}(t-s)^{q-1} y(s) d s .
$$

The detailed proof of Lemma 3.6 is similar to that of Lemma 3.1, so we omit it.

Proof of Theorem 3.2 The proof of Theorem 3.2 is similar to that of Theorem 3.1, we omit it. 
Theorem 3.3 Suppose that (A3) and (H1), (H2), (H3), and ( $\left.\mathrm{H}^{\prime \prime}\right)$ are satisfied, then there must be at least one solution of problem (1) in X provided that $2 T^{q}\|u\|_{\infty}+2 T^{q-1}\|v\|_{\infty}<$ $\Gamma(q)$.

To prove the theorem, we need the following lemmas.

Lemma 3.7 Assume that (A3) holds, then $L: \operatorname{dom} L \subset X \rightarrow Y$ is a Fredholm operator with index zero. And a linear continuous projector $P: X \rightarrow X$ can be defined by

$$
(P x)(t)=x(0)(1+k t)
$$

where $k=\frac{v_{1}}{v_{2}}=-\frac{v_{3}}{v_{4}}$. Furthermore, define the linear operator $K_{p} y: \operatorname{Im} L \rightarrow \operatorname{dom} L \cap \operatorname{ker} P$ as follows:

$$
\left(K_{p} y\right)(t)=\frac{1}{\Gamma(q)} \int_{0}^{t}(t-s)^{q-1} y(s) d s
$$

such that $K_{p}=\left(\left.L\right|_{\operatorname{dom} L \cap \operatorname{ker} P}\right)^{-1}$.

Proof Let $\varphi_{1}(t)=1+k t$. In view of $(A 3)$ we know

$$
\varphi_{1}(0)-\alpha I_{\eta}^{\gamma, \delta} \varphi_{1}(\zeta)=0, \quad \varphi_{1}(T)-\beta^{\rho} I^{p} \varphi_{1}(\xi)=0,
$$

we can easily get

$$
\operatorname{ker} L=\{c(1+k t): c \in \mathbb{R}\}
$$

Moreover, we can obtain that

$$
\operatorname{Im} L=\left\{y \in Y: v_{1}\left(\beta^{\rho} I^{p} J^{q} y(\xi)-J^{q} y(T)\right)=\alpha v_{3} I_{\eta}^{\gamma, \delta} J^{q} y(\zeta)\right\}
$$

We define the linear operator $P: X \rightarrow X$ as

$$
(P x)(t)=x(0)(1+k t)
$$

and the operator $Q: Y \rightarrow Y$ as

$$
(Q y)(t)=\left(v_{1}\left(\beta^{\rho} I^{p} J^{q} y(\xi)-J^{q} y(T)\right)-\alpha v_{3} I_{\eta}^{\gamma, \delta} J^{q} y(\zeta)\right) z_{2}(t),
$$

where $z_{2} \in Y$ satisfying $v_{2}\left(\beta^{\rho} I^{p} J^{q} z_{2}(\xi)-J^{q} z_{2}(T)\right)+\alpha v_{4} I_{\eta}^{\gamma, \delta} J^{q} z_{2}(\zeta)=1$.

In addition, for each $x \in \operatorname{dom} L \cap \operatorname{ker} P$, we have $x(0)=0$, then we get the generalized inverse operator of $L$ as follows:

$$
\begin{aligned}
\left(K_{p} y\right)(t) & =J^{q} y(t)-\frac{\alpha I_{\eta}^{\gamma, \delta} J^{q} y(\zeta)}{v_{2}} \cdot t \\
& =\frac{1}{\Gamma(q)} \int_{0}^{t}(t-s)^{q-1} y(s) d s-\frac{\alpha I_{\eta}^{\gamma, \delta} J^{q} y(\zeta)}{v_{2}} \cdot t .
\end{aligned}
$$

The detailed proof of Lemma 3.7 is similar to that of Lemma 3.1, so we omit it. 
Proof of Theorem 3.3 The proof of Theorem 3.3 is similar to that of Theorem 3.1, we omit it.

\author{
Acknowledgements \\ We would like to thank the referees very much for their valuable suggestions to improve this paper.
}

Funding

This work was partially supported by the Natural Science Foundation of China (11371221, 11571207, 51774197), the Shandong Natural Science Foundation (ZR2018MA011), and the Tai'shan Scholar Engineering Construction Fund of Shandong Province of China.

\title{
Competing interests
}

The authors declare that they have no competing interests.

\section{Authors' contributions}

The authors have made the same contribution. All authors read and approved the final manuscript.

\section{Author details}

${ }^{1}$ Department of Applied Mathematics, Shandong University of Science and Technology, Qingdao, P.R. China. ${ }^{2}$ State Key Laboratory of Mining Disaster Prevention and Control Co-founded by Shandong Province and the Ministry of Science and Technology, Shandong University of Science and Technology, Qingdao, P.R. China.

\section{Publisher's Note}

Springer Nature remains neutral with regard to jurisdictional claims in published maps and institutional affiliations.

Received: 2 December 2017 Accepted: 20 May 2018 Published online: 17 July 2018

\section{References}

1. Agarwal, R.P., Benchohra, M., Hamani, S.: A survey on existence results for boundary value problems of nonlinear fractional differential equations and inclusions. Acta Appl. Math. 109, 973-1033 (2010)

2. Ahmad, B., Ntouyas, S.K., Tariboon, J., Alsaedi, A.: A study of nonlinear fractional-order boundary value problem with nonlocal Erdélyi-Kober and generalized Riemann-Liouville type integral boundary conditions. Math. Model. Anal. 22(2), 121-139 (2017)

3. Ahmad, B., Ntouyas, S.K., Tariboon, J., Alsaedi, A.: Caputo type fractional differential equations with nonlocal Riemann-Liouville and Erdélyi-Kober type integral boundary conditions. Filomat 31(14), 4515-4529 (2017)

4. Bai, Z:: On positive solutions of a nonlocal fractional boundary value problem. Nonlinear Anal. 72, 916-924 (2010)

5. Bai, Z.: On solutions of some fractional $m$-point boundary value problems at resonance. Electron. J. Qual. Theory Differ. Equ. 2010, Article ID 37 (2010)

6. Bai, Z:: Solvability for a class of fractional $m$-point boundary value problem at resonance. Comput. Math. Appl. 62(3), 1292-1302 (2011)

7. Bai, Z., Chen, Y., Lian, H., Sun, S.: On the existence of blow up solutions for a class of fractional differential equations. Fract. Calc. Appl. Anal. 17, 1175-1187 (2014)

8. Cui, Y.: Solvability of second-order boundary-value problems at resonance involving integral conditions. Electron. J. Differ. Equ. 2012, Article ID 45 (2012)

9. Cui, Y.: Existence of solutions for coupled integral boundary value problem at resonance. Publ. Math. (Debr.) 89 73-88 (2016)

10. Cui, Y., Ma, W., Sun, Q., Su, X.: New uniqueness results for boundary value problem of fractional differential equation. Nonlinear Anal., Model. Control 23(1), 31-39 (2018)

11. Cui, Y., Ma, W., Wang, X., Su, X.: Uniqueness theorem of differential system with coupled integral boundary conditions. Electron. J. Qual. Theory Differ. Equ. 2018, Article ID 9 (2018)

12. Cui, Y., Sun, Q., Su, X.: Monotone iterative technique for nonlinear boundary value problems of fractional order $p \in(2,3]$. Adv. Differ. Equ. 2017, Article ID 248 (2017)

13. Cui, Y., Zou, Y.: Existence of solutions for second-order integral boundary value problems. Nonlinear Anal., Model. Control 21(6), 828-838 (2016)

14. Feng, W., Webb, J.R.L.: Solvability of three point boundary value problems at resonance. Nonlinear Anal. 30 3227-3238 (1997)

15. Graef, J.R., Kong, L.: Positive solutions for a class of higher order boundary value problems with fractional q-derivatives. Appl. Math. Comput. 218, 9682-9689 (2012)

16. Jiang, W., Kosmatov, N.: Solvability of a third-order differential equation with functional boundary conditions at resonance. Bound. Value Probl. 2017, Article ID 81 (2017)

17. Jiang, W., Qiu, J.: Solvability of $(k, n-k)$ conjugate boundary value problems at resonance. Electron. J. Differ. Equ. 2012, Article ID 114 (2012)

18. Kilbas, A.A., Srivastava, H.M., Trujillo, J.J.: Theory and Applications of Fractional Differential Equations. Elsevier, Amsterdam (2006)

19. Kosmatov, N., Jiang, W.: Second-order functional problems with a resonance of dimension one. Differ. Equ. Appl. 3, 349-365 (2016)

20. Kosmatov, N., Jiang, W.: Resonant functional problems of fractional order. Chaos Solitons Fractals 91, 573-579 (2016)

21. Liu, B., Li, J., Liu, L.: Existence and uniqueness for an $m$-point boundary value problem at resonance on infinite intervals. Comput. Math. Appl. 64, 1677-1690 (2012) 
22. Liu, L., Li, H., Liu, C., Wu, Y.: Existence and uniqueness of positive solutions for singular fractional differential systems with coupled integral boundary value problems. J. Nonlinear Sci. Appl. 10, 243-262 (2017)

23. Liu, L., Sun, F., Zhang, X., Wu, Y.: Bifurcation analysis for a singular differential system with two parameters via to degree theory. Nonlinear Anal., Model. Control 22(1), 31-50 (2017)

24. Liu, L., Zhang, X., Jiang, J., Wu, Y.: The unique solution of a class of sum mixed monotone operator equations and its application to fractional boundary value problems. J. Nonlinear Sci. Appl. 9(5), 2943-2958 (2016)

25. Liu, Y., Ge, W.: Solvability of nonlocal boundary-value problems for ordinary differential equations of higher order. Nonlinear Anal. 57, 435-458 (2004)

26. Mawhin, J.: Topological Degree Methods in Nonlinear Boundary-Value Problems. NSF CBMS Regional Conference Series in Mathematics. American Mathematical Society, Providence (1979)

27. Ntouyas, S.K., Tariboon, J.: Nonlocal boundary value problems for fractional differential inclusions with Erdélyi-Kober fractional integral boundary conditions. Science Asia 43(1), 47-55 (2017)

28. Qi, T., Liu, Y., Cui, Y.: Existence of solutions for a class of coupled fractional differential systems with nonlocal boundary conditions. J. Funct. Spaces 2017, Article ID 6703860 (2017)

29. Qi, T., Liu, Y., Zou, Y.: Existence result for a class of coupled fractional differential systems with integral boundary value conditions. J. Nonlinear Sci. Appl. 10, 4034-4045 (2017)

30. Sun, Q., Cui, Y.: Solvability of $(k, n-k)$ conjugate boundary value problems with integral boundary conditions at resonance. J. Funct. Spaces 2016, Article ID 3454879 (2016)

31. Sun, Q., Cui, Y.: Existence results for $(k, n-k)$ conjugate boundary-value problems with integral boundary conditions at resonance with $\operatorname{dim} \operatorname{ker} L=2$. Bound. Value Probl. 2017, Article ID 69 (2017)

32. Tian, Y., Bai, Z:: Existence results for the three-point impulsive boundary value problem involving fractional differential equations. Comput. Math. Appl. 59, 2601-2609 (2010)

33. Wang, Z:: A numerical method for delayed fractional-order differential equations: based on G-L definition. Appl. Math. Inf. Sci. 7, 525-529 (2013)

34. Zhang, S., Su, X.: The existence of a solution for a fractional differential equation with nonlinear boundary conditions considered using upper and lower solutions in reversed order. Comput. Math. Appl. 62, 1269-1274 (2011)

35. Zhang, X., Feng, M., Ge, W.: Existence result of second-order differential equations with integral boundary conditions at resonance. J. Math. Anal. Appl. 353, 311-319 (2009)

36. Zhang, X., Liu, L., Wu, Y.: The eigenvalue problem for a singular higher order fractional differential equation involving fractional derivatives. Appl. Math. Comput. 218, 8526-8536 (2012)

37. Zhang, X., Liu, L., Wu, Y.: Multiple positive solutions of a singular fractional differential equation with negatively perturbed term. Math. Comput. Model. 55, 1263-1274 (2012)

38. Zhang, X., Liu, L., Wu, Y.: The uniqueness of positive solution for a fractional order model of turbulent flow in a porous medium. Appl. Math. Lett. 37, 26-33 (2014)

39. Zhang, X., Liu, L., Wu, Y., Cui, Y.: Entire blow-up solutions for a quasilinear pLaplacian Schrödinger equation with a non-square diffusion term. Appl. Math. Lett. 74, 85-93 (2017)

40. Zhao, Z., Liang, J.: Existence of solutions to functional boundary-value problem of second-order nonlinear differential equation. J. Math. Anal. Appl. 373, 614-634 (2011)

41. Zou, Y., Cui, Y.: Existence results for a functional boundary value problem of fractional differential equations. Adv. Differ. Equ. 2013, Article ID 233 (2013)

42. Zou, Y., He, G.: On the uniqueness of solutions for a class of fractional differential equations. Appl. Math. Lett. 74 68-73 (2017)

43. Zou, Y., Liu, L., Cui, Y.: The existence of solutions for four-point coupled boundary value problems of fractional differential equations at resonance. Abstr. Appl. Anal. 2014, Article ID 314083 (2014)

\section{Submit your manuscript to a SpringerOpen ${ }^{\circ}$ journal and benefit from:}

- Convenient online submission

- Rigorous peer review

- Open access: articles freely available online

- High visibility within the field

- Retaining the copyright to your article

Submit your next manuscript at $\gg$ springeropen.com 